Methods 64 CHF patients were randomised equally into two groups. All were treated with the basic conventional treatment. Additionally, $1.2 \mathrm{~g}$ of $\mathrm{OC}$ was given three times a day to the therapy group, while no additional treatment was given to the control group. During the treatment, body weight, urine output, sweat secretion and $6 \mathrm{~min}$ walking distance were observed in the two groups. The dosage of cedilanid and furosemide for the first week and second week in the two groups was analysed. The levels of serum $\mathrm{Na}^{+}$in two groups were respectively measured on the second, eighth and fifteenth hospital day. During the treatment, the sideeffect and compliance were observed.

Results 1 and 2 weeks after treatment, the number of sweat secretion was less in two groups, but the decrement was more significant in the therapy group than in the control group $(p<0.05)$. One weeks later, the urine output was more than that before $(p<0.05)$, body weight became less $(p<0.05)$, and 6 min walking distance was better than before, but there are all no significant differences between two groups ( $p>0.05) .2$ weeks later, in both groups, the urine output was more than that before; body weight became less; 6 minwalking distance was better than before. All these differences are significant differences statistically $(p<0.05)$. And there are significant differences about urine output and 6 min walking distance between two groups statistically $(\mathrm{p}<0.05)$; about body weight between two groups there is no significant difference $(p>0.05)$. There are no significant differences about the dosage of lanatoside $\mathrm{C}$ and furosemide in the first and second week between two groups statistically ( $p>0.05)$. The level of serum $\mathrm{Na}^{+}$on the eighth hospital day was less than before in both groups $(p<0.05)$, but there are no significant differences between two groups statistically ( $p>0.05$ ); on the fifteenth day, there is almost no change about that of the therapy group compared with that on the eighth day $(p>0.05)$; on the contrary, there is a significant difference in the control group statistically $(p<0.05)$; and there is a significant difference between two groups statistically $(p<0.05)$. During the period of using the capsule, the 33 patients have not any uncomfortable complain about this drug and nobody stop using this capsule.

Conclusion The effect of $\mathrm{OC}$ about releasing hyperidrosis in a short time is conspicuous. It can play the role in a short time. There is no obvious effect on body weight. The short-term effects of $\mathrm{QC}$ about improving urine output and $6 \mathrm{~min}$ walking distance are not significant, but it plays this role during the second week gradually. QC can't reduce the dosage of cedilanid and furosemide. OC has the effect of keeping the level of serum $\mathrm{Na}^{+}$to some extent. During the observation, the tolerance of this capsule is good, and there is no side effect observed.

\section{e0334 INVESTIGATION FOR EFFECTS OF SOTALOL COMBINED WITH PROPAFENONE TO TREAT PAEDIATRIC ATRIAL TACHYCARDIA}

doi:10.1136/hrt.2010.208967.334

Li Xiaomei, Zhang Yan, Zhang Yi, Bao Min, Lu Ping. First Hospital of Tsinghua University pediatric Cardiac Department

Objects To evaluate the efficacy, safety, selection and combination program of anti-arrhythmic drugs to treat paediatric atrial tachycardia.

Methods 42 children (male 19, female 23, age 7 days-14 years) diagnosed of atrial tachycardia were treated by oral anti-arrhythmic drugs. $24 \mathrm{~h}$ holter monitoring and wireless remote realtime monitoring system were used to evaluate the efficacy. Cardiac function was evaluated by echocardiogram. Side effects of drugs were closely detected during the whole process.

Results 21 cases were firstly treated by single sotalol, none were completely cured, 20 were partially cured (95.2\%), 1 was ineffective
(4.8\%). 41 cases were finally treated by sotalol plus propafenone, 30 were completely cured (73.2\%), 9 were partially cured $(21.9 \%)$ and 2 were ineffective $(4.9 \%)$. The effective dose of sotalol was $5.4-5.7 \mathrm{mg} / \mathrm{kg} . \mathrm{d}$, and the effective dose of propafenone was 9-10.5 mg/kg.d. 5 cases of incessant atrial tachycardia complicated by tachycardiomyopathy $(5 / 17,29.4 \%)$ were treated by amiodarone plus metroprolol, 1 was completely cured and the others' cardiac function were obviously improved after treatment although none of them were completely cured, they all reverted to sotalol plus propafenone while cardiac function became nearly normal. Injury of thyroid function happened in 3 cases after taking oral amiodarone for 3-8 months and all recovered during 1 month after withdrawing the drug. During the 1-14 months of follow up for the 31 cases completely cured, only 1 case of intermittent atrial tachycardia recurred at No.8 month after withdrawing the drug.

Conclusion 1. Sotalol is a safe and effective anti-arrhythmic drug which can be used to treat paediatric atrial tachycardia, effective dose is $4.89-5.71 \mathrm{mg} / \mathrm{kg} . \mathrm{d}$; 2 . Combination use of sotalol plus propafenone is obviously more effective than single use of sotalol with satisfactory safety; 3 . Combination use of sotalol plus propafenone is safer and more effective than combination use of amiodarone plus metroprolol. 4. Amiodarone might lead to thyroid function injury, and can be used as transitional drug for patients diagnosed of tachycardiomyopathy.

\section{e0335 ROLE OF OT INTERVAL DYNAMICITY IN PREDICTING SUDDEN DEATH IN PATIENTS WITH IDIOPATHIC DILATED CARDIOMYOPATHY}

doi:10.1136/hrt.2010.208967.335

Mingwei Bao, Tuantuan Tan, Shengbo Yu, Kui Chen, Congxin Huang. Renmin Hospital of Wuhan University

Objective To investigate the role of OT interval dynamicity in predicting sudden death in patients with idiopathic dilated cardiomyopathy (DCM).

Methods 55 patients with DCM (DCM group) and 27 health people (Control group, Con) were enrolled according to certain criteria. Investigations included history collection, clinical examination, echocardiography, ECG and $24 \mathrm{~h}$ ambulatory ECG. Following indexes were determined, left ventricle end diastolic dimension (LVEDD), left ventricle ejection fraction (LVEF), OT dispersion (QTd), SDNN, the slope of OT/RR plots of the linear regression, ventricular premature beats (VPB) and non-sustained ventricular tachycardia (NSVT). Patients with DCM were followed-up until all cause death.

Results Comparing with Con group, the LVEDD, OTd, VPB/24 h, NSVT/24 h, QTe/RR slope and OTp/RR slope were higher in DCM group, while LVEF and SDNN were lower in DCM group $(p<0.05)$. The LVEDD, LVEF, OTd, SDNN, OTe/RR slope and OTp/RR slope were significant different among DCM sudden death group, DCM non sudden death group and Con group $(\mathrm{p}<0.05)$. Comparing between DCM sudden death and non sudden death group, LVEF, SDNN, QTp/RR slope and OTp/RR slope shown significant difference $(p<0.05)$. Comparing between DCM with NSVT and without NSVT group, LVEF, OTd, VPB/24 h, OTp/RR slope and QTp/RR slope shown significant difference $(p<0.05)$. The sudden death rate of DCM patients with $\mathrm{QTe} / \mathrm{RR}$ slope $\geq 0.210$ was higher than those $<0.210$ ( $54.5 \%$ vs $21.1 \%, \mathrm{p}<0.05)$. Sudden death rate of QTp/RR slope $\geq 0.190$ was higher than those $<0.190(52.2 \%$ vs $21.9 \%, \mathrm{p}<0.05)$. The sudden death rate of DCM patients with both LVEF $35 \%$ and NSVT+ was $62.5 \%$. Combining $\mathrm{OTe} / \mathrm{RR} \geq 0.210$ with NSVT+ or LVEF $35 \%$, the sudden death rates were $62.5 \%$ or $66.7 \%$. Combining QTp/RR $\geq 0.190$ with NSVT + or LVEF $35 \%$, the sudden death rates were $66.7 \%$ or $61.5 \%$. Combining QTe/ $\mathrm{RR} \geq 0.210$ or $\mathrm{QTp} / \mathrm{RR} \geq 0.190$ with NVST+ and LVEF $35 \%$, the sudden death rates were $77.8 \%$ or $70.0 \%$. 